\title{
Grating-Graphene Metamaterial as a Platform for Terahertz Nonlinear Photonics
}

\author{
Jan-Christoph Deinert, David Alcaraz Iranzo, Raúl Pérez, Xiaoyu Jia, Hassan A. Hafez, Igor Ilyakov, \\ Nilesh Awari, Min Chen, Mohammed Bawatna, Alexey N. Ponomaryov, Semyon Germanskiy, \\ Mischa Bonn, Frank H.L. Koppens, Dmitry Turchinovich, Michael Gensch, Sergey Kovalev,* \\ and Klaas-Jan Tielrooij*
}

Cite This: ACS Nano 2021, 15, 1145-1154

Read Online

\section{ACCESS |}

山lll Metrics \& More

回 Article Recommendations

Supporting Information

ABSTRACT: Nonlinear optics is an increasingly important field for scientific and technological applications, owing to its relevance and potential for optical and optoelectronic technologies. Currently, there is an active search for suitable nonlinear material systems with efficient conversion and a small material footprint. Ideally, the material system should allow for chip integration and room-temperature operation. Two-dimensional materials are highly interesting in this regard. Particularly promising is graphene, which has demonstrated an exceptionally large nonlinearity in the terahertz regime. Yet, the light-matter interaction length in two-dimensional materials is inherently minimal, thus limiting the overall nonlinear optical conversion
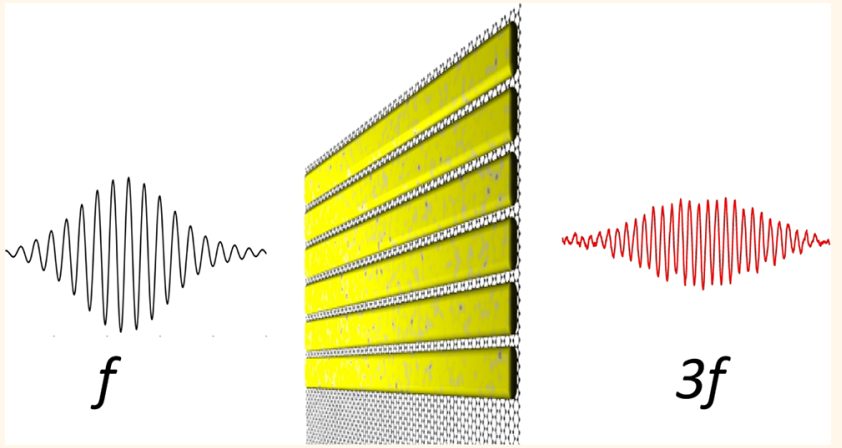
efficiency. Here, we overcome this challenge using a metamaterial platform that combines graphene with a photonic grating structure providing field enhancement. We measure terahertz third-harmonic generation in this metamaterial and obtain an effective third-order nonlinear susceptibility with a magnitude as large as $3 \times 10^{-8} \mathrm{~m}^{2} / \mathrm{V}^{2}$, or $21 \mathrm{esu}$, for a fundamental frequency of $0.7 \mathrm{THz}$. This nonlinearity is 50 times larger than what we obtain for graphene without grating. Such an enhancement corresponds to a third-harmonic signal with an intensity that is 3 orders of magnitude larger due to the grating. Moreover, we demonstrate a field conversion efficiency for the third harmonic of up to $\sim 1 \%$ using a moderate field strength of $\sim 30 \mathrm{kV} / \mathrm{cm}$. Finally, we show that harmonics beyond the third are enhanced even more strongly, allowing us to observe signatures of up to the ninth harmonic. Grating-graphene metamaterials thus constitute an outstanding platform for commercially viable, CMOS-compatible, room-temperature, chipintegrated, $\mathrm{THz}$ nonlinear conversion applications.

KEYWORDS: terahertz, harmonics, graphene, nonlinear, field enhancement, metamaterial

$\mathrm{T}$ he ability to perform nonlinear optical conversion has relevance for a broad range of current and future technologies, including ultrashort pulse generation, advanced spectroscopy, optical information processing and storage, telecommunications, light harvesting, bioimaging, integrated optics, and quantum technologies. ${ }^{1-4}$ For many of these applications, it is crucial to find nonlinear optical material systems that allow for integration into existing nanophotonic platforms and for operation with low power consumption. Thus, ideal nonlinear optical materials should be compatible with complementary metal-oxide-semiconductor (CMOS) technology, have a small material footprint, and have a large nonlinear susceptibility, such that large nonlinear effects occur for small incident optical fields. Furthermore, they should ideally operate at room temperature. Steady progress has been made in recent years, with suitable material systems operating in the visible and near-infared parts of the electromagnetic spectrum, for example, using indium tin oxide with a thickness of tens of nanometers. ${ }^{5,6}$ However, currently, no commercially viable nonlinear material system is available for incident light in the terahertz region (roughly $0.3-30 \mathrm{THz}$ ) of the electromagnetic spectrum. This technological gap needs to be filled because the $\mathrm{THz}$ region holds great promise for both

Received: September 25, 2020

Accepted: November 25, 2020

Published: December 11, 2020 


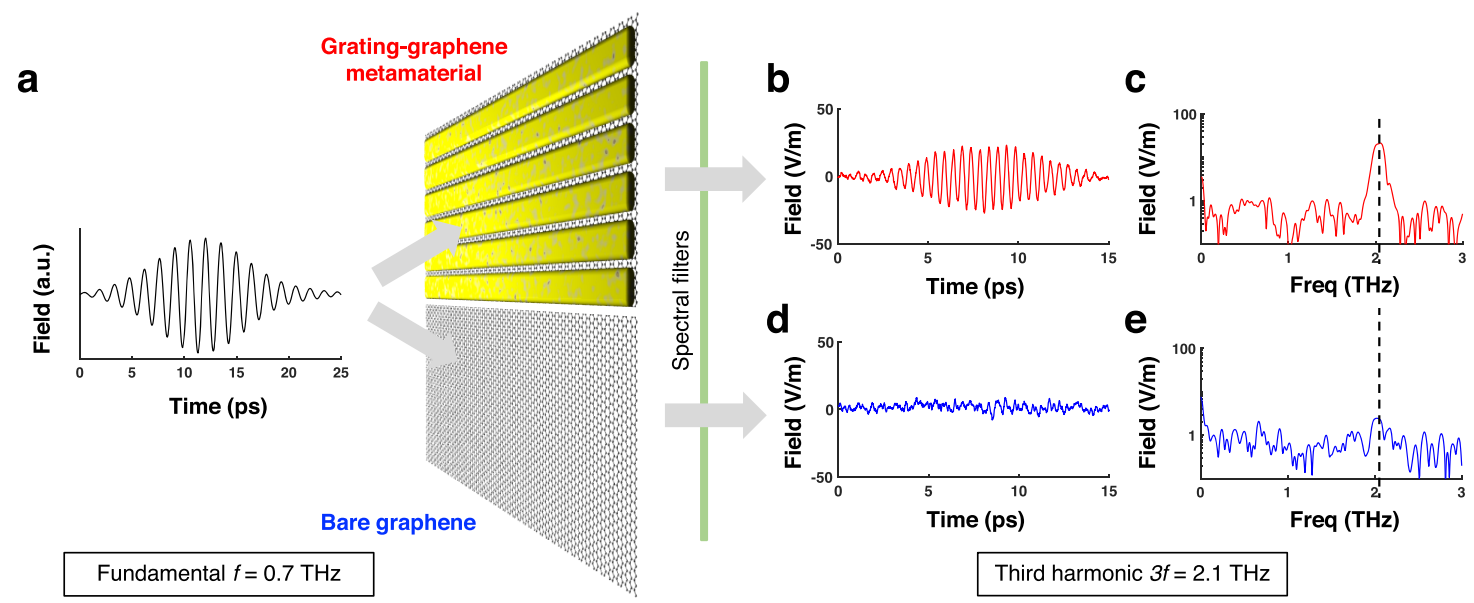

Figure 1. Enhanced third-harmonic generation in grating-graphene metamaterial. (a) Schematic representation of the measurement configuration, with a multicycle THz waveform with fundamental frequency $f$ incident on a grating-graphene metamaterial sample (top) or a bare graphene sample (bottom). In the metamaterial, field enhancement occurs inside the metal gap, which leads to nonlinearly enhanced generation of third-harmonic signal. (b,c) Measured THz field strength in the time (b) and frequency (c) domain for the grating-graphene metamaterial, with an incident field strength of $12 \mathrm{kV} / \mathrm{cm}$. (d,e) Measured THz field strength in the time (d) and frequency (e) domain for the bare graphene sample with an incident field strength of $13.6 \mathrm{kV} / \mathrm{cm}$. Clearly, a significantly more intense harmonic signal is created in the graphene metamaterial sample.

scientific and technological applications, such as imaging, industrial quality control, gas sensing, and wireless communications. All of the above would benefit tremendously from the availability of integrated nonlinear optical devices in the $\mathrm{THz}$ range.

It is particularly appealing to use nonlinear optical materials that are thinner than the wavelength of light. In this case, different optical waves propagating through the material automatically remain in phase, thus removing the need for complex engineering to achieve phase matching. As a result, nonlinear optical conversion in ultimately thin, two-dimensional materials has received ample attention, as reviewed recently in ref 7 . However, the challenge associated with using subwavelength materials is that-even with extraordinary large nonlinear coefficients-there is simply not much material to interact with, thus usually leading to low nonlinear conversion efficiencies. A promising strategy to overcome this challenge had already been identified before the advent of twodimensional materials. Namely, photonic structures can be used to enhance light-matter interactions and thereby increase the nonlinear conversion efficiency. Typical photonic structures that have been used include nanoparticles, ${ }^{8}$ hole arrays, ${ }^{9,10}$ nanocavity gratings, ${ }^{11}$ nanocavity-antenna systems, $^{12}$ split-ring resonators, ${ }^{13,14}$ and nanoantennas. ${ }^{4,6,15}$ Combining a photonic structure with a nonlinear material has, for example, made it possible to obtain a surface-enhanced Raman spectrum of a single molecule ${ }^{8}$ and to observe thirdharmonic generation from a single semiconductor nanoparticle. $^{4}$

Nonlinear effects in two-dimensional materials are particularly strong for light in the $\mathrm{THz}$ region. Indeed, twodimensional, gapless Dirac materials, where electrons obey a linear energy-momentum dispersion relation, have shown very large nonlinear coefficients in the $\mathrm{THz}$ range. In particular, $\mathrm{THz}$ third-harmonic generation has been observed using monolayer graphene, ${ }^{16}$ using the Dirac surface states in the topological insulator $\mathrm{Bi}_{2} \mathrm{Se}_{3},{ }^{1,18}$ and using the Dirac semimetal $\mathrm{Cd}_{3} \mathrm{As}_{2}{ }^{19,20}$ The $\mathrm{THz}$ third-order nonlinear susceptibility of graphene was found to be especially large: $\left|\chi^{(3)}\right| \approx 10^{-9} \mathrm{~m}^{2} /$ $\mathrm{V}^{2}{ }^{16}$ This value is many orders of magnitude larger than its $\chi^{(3)}$ in the near-infrared range, which is below $\sim 10^{-16} \mathrm{~m}^{2} / \mathrm{V}^{2} .{ }^{21}$ The main reason for the strong $\mathrm{THz}$ nonlinearity is that it is the result of a different nonlinearity mechanism than the one in the near-infrared. Namely, the nonlinearity originates from the collective thermodynamic response of the quasi-free carriers in graphene. Their response to an incident $\mathrm{THz}$ pulse can be outlined as follows (see also refs 16, 22, and 23): as a first step, graphene conduction-band electrons absorb the energy from incident $\mathrm{THz}$ light through Drude absorption. This energy is then distributed among all electrons, leading to a thermal quasi-equilibrium within the electronic system, as it has not yet thermalized with the lattice. This is because the $\mathrm{THz}$ oscillation period and the electron cooling time are on the order of a picosecond and thus much longer than the electron thermalization time, which is below 100 fs. The electronic temperature increase of the quasi-equilibrium Fermi-Dirac distribution is substantial due to the small electronic heat capacity of graphene. ${ }^{24}$ Since the $\mathrm{THz}$ conductivity-and therefore $\mathrm{THz}$ absorption-decreases for increasing electron temperature (and Fermi energy above $\sim 0.1 \mathrm{eV}$ ), ${ }^{2,25,26}$ this gives rise to a large, ultrafast thermodynamic $\mathrm{THz}$ nonlinearity. Quasi-monochromatic $\mathrm{THz}$ excitation leads to ultrafast modulation of the graphene $\mathrm{THz}$ conductivity, and hence of the driven $\mathrm{THz}$ currents, within each cycle of the driving $\mathrm{THz}$ field due to the electronic heating-cooling dynamics. This, in turn, leads to a nonlinear response that manifests as harmonic generation at odd-order multiples of the frequency of the driving field. Given this strong nonlinearity and the fact that graphene can be integrated with CMOS-based chips, as demonstrated in ref 27 , it is a highly promising candidate material for commercially viable, chip-integrated, $\mathrm{THz}$ nonlinear conversion applications. One important hurdle, however, that needs to be overcome is to ensure sufficient light-matter interaction between incident $\mathrm{THz}$ light and a monolayer thin material. 

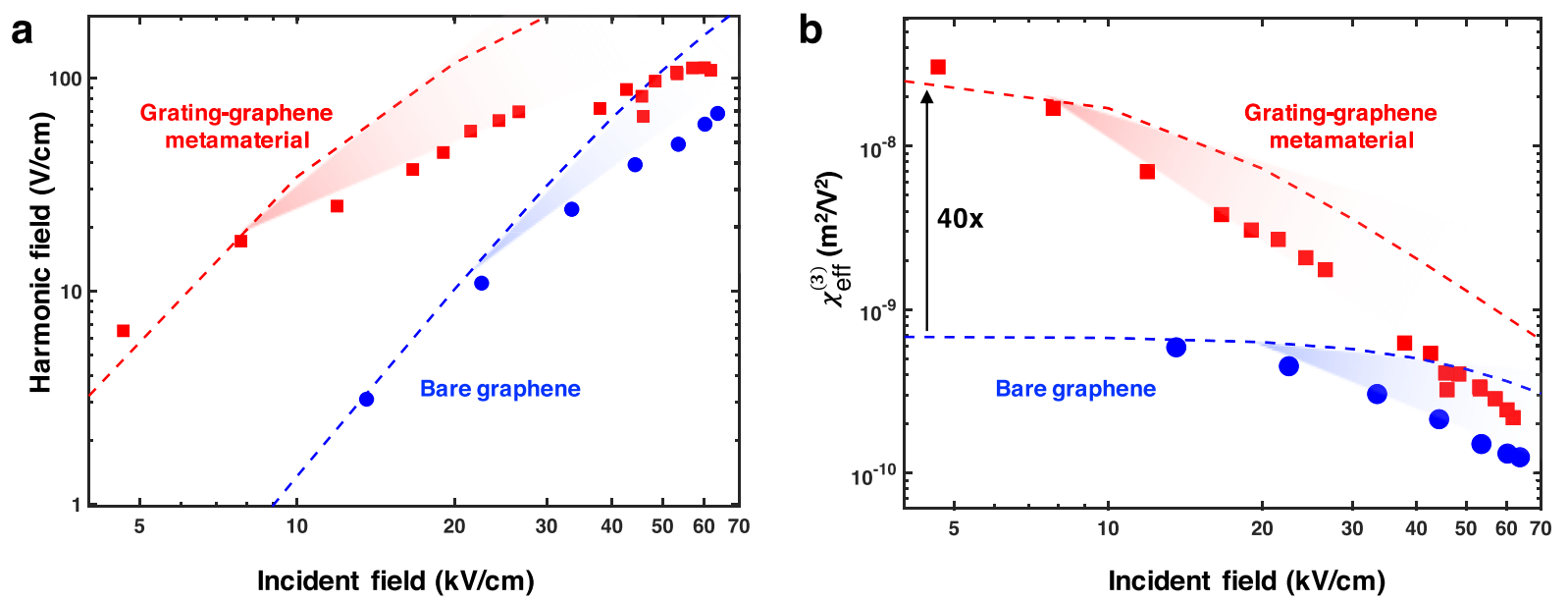

Figure 2. Nonlinearity of grating-graphene metamaterial $v s$ bare graphene. (a) Comparison of third-harmonic intensity for grating-graphene metamaterial sample A (red squares) and bare graphene sample B (blue circles), as a function of peak field strength of the incident THz light. The blue dashed line is the result of split-step simulations based on thermodynamic nonlinearity without field enhancement. The red dashed line represents the same simulation, now with field-enhancement factor $M=5$ and active area $(1-\eta)=10 \%$. (b) Extracted thirdorder nonlinear susceptibility $\chi_{\text {eff }}^{(3)}$ as a function of incident peak field strength for grating-graphene metamaterial sample A (red squares) and bare graphene sample B (blue circles). The dashed lines show the results of the thermodynamic model. The decreasing trends with incident field strength indicate the occurrence of saturation effects. For low field strengths, the metal grating leads to a factor 40-50 increase in $\chi_{\text {eff }}^{(3)}$, corresponding to more than 3 orders of magnitude enhanced harmonic intensity. The red and blue shaded regions in both panels indicate the onset of saturation effects not captured by the model.

In this work, we demonstrate a metamaterial system consisting of graphene and a metallic grating, where the grating provides field enhancement, thus leading to a strong increase of the $\mathrm{THz}$ nonlinear light-matter interaction. We examine the generation of odd $\mathrm{THz}$ harmonics and observe that the third-harmonic intensity can be enhanced by more than 3 orders of magnitude due to the integration of the metal grating. We discuss quantitatively how this enhancement is achieved and what the limitations of this approach are and the perspectives for further enhancing $\mathrm{THz}$ nonlinearities with this photonic approach. Finally, we show a field conversion efficiency of $1 \%$ and signatures of higher harmonics, including the ninth harmonic, using a moderate incident field strength of $\sim 30 \mathrm{kV} / \mathrm{cm}$.

\section{RESULTS AND DISCUSSION}

Grating-Enhanced THz Third-Order Nonlinearity. A natural and proven method to study the nonlinear properties of a material is to quantify the intensity or field strength of generated harmonic signal compared to the intensity or field strength of incident fundamental light. This method has already been applied successfully to graphene, ${ }^{16}$ and therefore, we follow the same experimental procedure. Briefly (see Methods and Supporting Information for details), this method consists of focusing a narrow-band $\mathrm{THz}$ waveform, generated by the accelerator-based TELBE beam facility, ${ }^{28}$ onto a sample and measuring the electric field of the transmitted waveform as a function of time through electro-optic sampling. In Figure 1a, we show a measured incident $\mathrm{THz}$ waveform with fundamental frequency $f=0.7 \mathrm{THz}$ in the time domain, together with a schematic of a grating-graphene metamaterial sample and a bare graphene sample. Figure $1 \mathrm{~b}$ shows the signal in the time domain after passing through the metamaterial sample and spectral filters (two band-pass filters at $2.1 \mathrm{THz}$ ). These bandpass filters suppress spectral components at the fundamental frequency, while transmitting the third-harmonic signal, in order to be able to resolve this signal well within the dynamic range of the detectors. Clearly, the measured oscillations are faster than those of the incident light. The Fourier transform (see Methods and Supporting Information) in Figure 1c shows that these fast oscillations correspond to the generated third harmonic at $3 f=2.1 \mathrm{THz}$.

We first compare the nonlinear conversion efficiency of two samples, both consisting of wet-transferred monolayer graphene on quartz with an estimated Fermi energy of $\sim 0.23$ $\mathrm{eV}$ (see Methods and Supporting Information for details). Sample A has a gold metallic grating on top of the graphene, separated by a $2 \mathrm{~nm}$ thick layer of $\mathrm{Al}_{2} \mathrm{O}_{3}$. The metal grating contains metallic stripes with a metal width of $w_{\text {metal }}=18 \mu \mathrm{m}$, separated by gaps with a width of $w_{\text {gap }}=2 \mu \mathrm{m}$. This corresponds to a duty cycle $\eta=\frac{w_{\text {metal }}}{w_{\text {metal }}+w_{\text {gap }}}$ of $90 \%$. Thus, $90 \%$ of the graphene area is covered by metal, and there is $10 \%$ active graphene area. This duty cycle is the parameter that governs the field enhancement and absorption in the gratinggraphene metamaterial samples, as we will show. Sample B also contains $2 \mathrm{~nm}$ of $\mathrm{Al}_{2} \mathrm{O}_{3}$ on top of graphene, while not containing a metal grating. As a first indication that our approach of using a grating-graphene metamaterial to enhance nonlinear conversion works, we use a small incident field strength of $E_{\mathrm{f}}=12 \mathrm{kV} / \mathrm{cm}$ and observe clear third-harmonic signal for the grating-graphene metamaterial sample A (Figure $1 \mathrm{~b}, \mathrm{c})$. However, for the bare graphene sample B with a slightly larger incident field strength of $14 \mathrm{kV} / \mathrm{cm}$, there is no clearly observable third-harmonic signal in the time trace (see Figure 1d). In the Fourier spectrum (see Figure 1e), we observe a small signal just above the noise floor. Clearly, the metamaterial sample has a much stronger nonlinearity, even though only $10 \%$ of the graphene is part of the active area. We note that the metallic grating itself does not absorb much $\mathrm{THz}$ light and does not generate any significant third-harmonic signal (see Supporting Information). 
In order to quantify the nonlinear properties of our gratinggraphene metamaterial, we compare how the field strength of the third-harmonic signal $E_{3 \mathrm{f}}$ scales with the incident field strength $E_{\mathrm{f}}$ (see Figure $2 \mathrm{a}$ ). These data points are obtained by taking the Fourier transform of transmitted time traces as in Figure 1 and taking the peak of the power spectrum around the frequency of the third-harmonic peak at $2.1 \mathrm{THz}$. We observe that, for the lowest field strengths, $E_{3 \mathrm{f}}$ is more than an order of magnitude higher in the metamaterial sample than in bare graphene, whereas the third-harmonic signals are more similar for the two samples at the highest field strengths. We extract the third-order susceptibility using ${ }^{16}$

$$
\left|\chi_{\text {eff }}^{(3)}\right|=\frac{E_{3 f}}{E_{\mathrm{f}}^{3}} \frac{4 c \cdot n_{3 \mathrm{f}}}{3 \pi f d}
$$

where $c$ is the speed of light and $d=0.3 \mathrm{~nm}$ is the thickness of graphene. For the refractive index of graphene at the thirdharmonic frequency, we conservatively use $n_{3 \mathrm{f}}=10$, as in ref 16. This value can be up to an order of magnitude larger, which would make the nonlinear coefficient an order of magnitude larger. The results in Figure $2 \mathrm{~b}$ show that for low incident field strength the grating-graphene metamaterial reaches an experimental value of $\left|\chi_{\text {eff }}^{(3)}\right|=3 \times 10^{-8} \mathrm{~m}^{2} / \mathrm{V}^{2}$ (using $n_{3 \mathrm{f}}=$ $10)$. In esu units, this corresponds to 29 $\left|\chi_{\text {eff }}^{(3)}\right|[\mathrm{esu}]=\frac{9 \times 10^{9}}{4 \pi}\left|\chi_{\text {eff }}^{(3)}\right|=21$ esu. Expressed as a surface nonlinearity, we obtain $\left|\chi_{2 \mathrm{D}}^{(3)}\right|=\left|\chi_{\text {eff }}^{(3)}\right| \cdot d=10^{-17} \mathrm{~m}^{3} / \mathrm{V}^{2}$. We can also express the nonlinearity as the nonlinear refractive index $n_{2}$, where the intensity-dependent refractive index is given as ${ }^{1} n=n_{0}+n_{2} \times I_{\mathrm{THz}}$. Here $n_{0}$ is the linear refractive index and $I_{\mathrm{THz}}$ is the intensity of the incoming $\mathrm{THz}$ light. We estimate an $n_{2}$ well above $100 \mathrm{~cm}^{2} / \mathrm{GW}$ (see Methods). Finally, we note that the sign of the $\mathrm{THz}$ nonlinearity, and therefore of $\chi_{\mathrm{eff}}^{(3)}$ and $n_{2}$, is negative, as the refractive index decreases upon $\mathrm{THz}$ excitation (see also ref 16), in agreement with the sign of the nonlinearity in the near-infrared region. ${ }^{30}$

Comparing the nonlinear susceptibility of the gratinggraphene metamaterial sample A with graphene without grating (sample B), we find a value that is $\sim 50$ times larger. The obtained value of $\left|\chi_{\text {eff }}^{(3)}\right|$ is also more than 3 orders of magnitude larger than the highest $\mathrm{THz}$ nonlinearities reported for quantum well systems. ${ }^{31-33}$ We can also compare the $\mathrm{THz}$ nonlinearity of our grating-graphene metamaterial with the near-infared nonlinearities found very recently for thin films of indium tin oxide in a special frequency region with epsilon near zero, with a nonlinear refractive index $n_{2}$ of $\sim 0.1 \mathrm{~cm}^{2} / \mathrm{GW}$ without field enhancement ${ }^{5}$ and $\sim 4 \mathrm{~cm}^{2} / \mathrm{GW}$ with nanoantenna-induced enhancement. ${ }^{6}$ These nonlinear refractive indices are lower than what we find for our grating-graphene metamaterial in the $\mathrm{THz}$ regime. Overall, we can conclude that the $\mathrm{THz}$ nonlinearity of our grating-graphene metamaterial is large, compared to any nonlinear optical (meta)material at any wavelength, operating at room temperature.

We can understand the enhancement of the nonlinearity induced by the metal grating quantitatively by incorporating field enhancement into the thermodynamic model of refs 16 and 22. For bare graphene sample B, we straightforwardly use the incident field strength $E_{f}$, the modal carrier density obtained from Raman measurements (see Supporting Information), an estimated momentum scattering time, and standard picosecond cooling dynamics as the input parameters for the simulation. This produces the blue dashed lines in Figure 2, which describe the experimental data quite well up to an incident field strength of $\sim 20 \mathrm{kV} / \mathrm{cm}$. For higher field strengths, there is more saturation in the experimental data than what is predicted by the model. We will discuss saturation effects in more detail in the next section. For the simulations of metamaterial sample $A$, we use the exact same input parameters as for sample $B$, with the difference that we use as incident field strength $M \times E_{f}$, where $M$ is the fieldenhancement factor. We estimate an average field-enhancement factor $M$ of $\sim 5$ inside the gap of our grating, using rigorous coupled wave analysis (RCWA), following ref 34 (see Supporting Information). The resulting third-harmonic signal would correspond to a sample where the whole graphene area experiences 5-fold field enhancement, whereas in our case, the active area is only $1-\eta=10 \%$. Therefore, we multiply the simulation results with a factor $\sqrt{1-\eta}$, where the square root comes from the fact that we consider field rather than intensity. The results are the red dashed lines in Figure 2, which match the data for the lowest field strengths. Thus, the experimental results are in agreement with a grating-induced field enhancement of a factor $\sim 5$.

Importantly, the third-harmonic signal is increased in a nonlinear fashion by the field enhancement. Indeed, we find that for the lowest incident field strengths, the model predicts an increase of the third-harmonic field strength by a factor $\sim 40$, corresponding to an intensity enhancement factor of $>1000$. We can understand these numbers analytically, as for the $n$th harmonic, we expect

$$
\frac{E_{\mathrm{nf}}^{\text {(grating) }}}{E_{\mathrm{nf}}^{\text {(nograting) }}}=M^{n} \cdot \sqrt{1-\eta}
$$

For $n=3, M=5$, and $\eta=90 \%$, the analytical model gives the same factor of $\sim 40$ as the numerical model based on the thermodynamic nonlinearity. This factor is in good agreement with the experimental observation of a $\left|\chi_{\text {eff }}^{(3)}\right|$ that is $\sim 50$ times larger for the grating-graphene metamaterial sample $\mathrm{A}$, in comparison with the bare graphene sample $\mathrm{B}$. The small discrepancy could come from a larger field-enhancement factor $M$ or from the fact that neither the thermodynamic nor the analytical model take into account that the grating could also lead to more efficient out-coupling of harmonic light to the farfield, where it is detected. We note that in the graphene-filled gap region, and in the perturbative low-field regime, the thirdharmonic intensity is enhanced by a factor $M^{6}$ (see eq 1 ), which is more than 4 orders of magnitude.

Saturation of Harmonic Generation. We now discuss the occurrence of saturation effects, which follow clearly from Figure $2 b$, where without saturation one would obtain a flat line. We first discuss two saturation mechanisms that are captured by the thermodynamic model. The first reason for saturation is the occurrence of saturable absorption: if the carrier temperature increases, the graphene conductivity decreases and the absorption of $\mathrm{THz}$ light is reduced (see also Supporting Information). Eventually, the carrier temperature can become so high that virtually no incident $\mathrm{THz}$ light is absorbed, as shown in ref 22. The second reason that saturation occurs is that-with constant absorption-the increase in carrier temperature of graphene scales sublinearly with the intensity of the incident light, which is the result of the electronic heat capacity scaling with temperature. ${ }^{35}$ This 


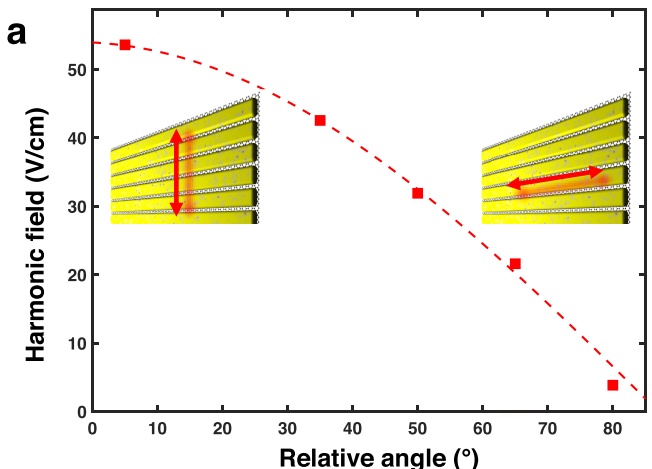

b
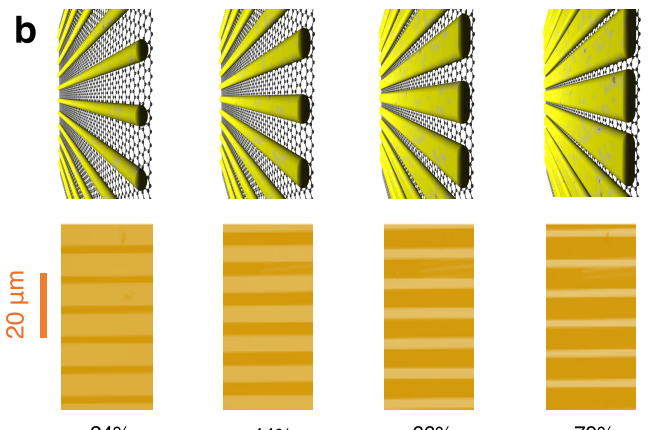

$66 \%$

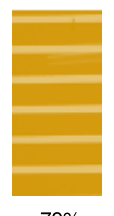

$79 \%$

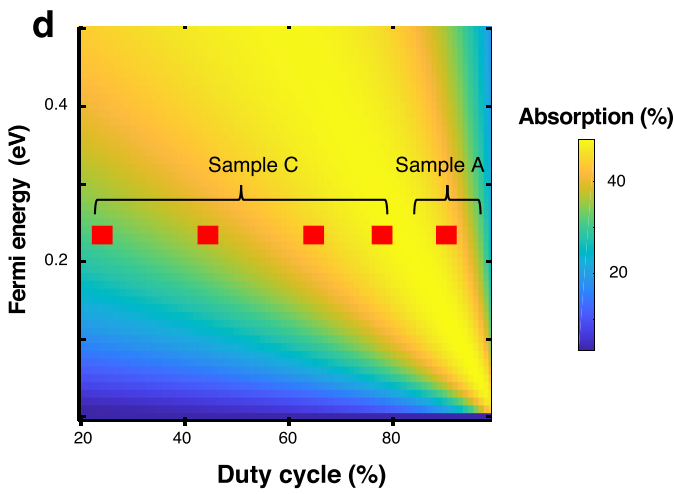

Figure 3. Effect of grating orientation and duty cycle. (a) Field strength of the created third-harmonic signal as a function of orientation of the metal grating with respect to the polarization of the incoming fundamental THz light (red squares; sample A; incident field strength of $\sim 32 \mathrm{kV} / \mathrm{cm}$ ), as illustrated in the schematic insets. The dashed line is a $\sin ^{2}$ function to guide the eye. (b) Schematic representation (top) and optical images (bottom) of the four different grating-graphene areas of sample C, with duty cycles of $24,44,66$, and $79 \%$. Metal and gap widths are to scale. (c) Field strength of the created third-harmonic signal as a function of duty cycle using sample $\mathrm{C}$ (red squares; incident peak field strength of $\sim 14 \mathrm{kV} / \mathrm{cm}$ ), showing an increase, followed by saturation, for increasing duty cycle. The thermodynamic model (dashed line) matches the experimental results for the lowest duty cycles before saturation effects occur, as indicated by the red shaded region. The inset shows the absorption of fundamental light $v s$ duty cycle, showing larger absorption at higher duty cycle. This is obtained using ( 1 - transmitted fundamental intensity) and multiplying this by a constant, such that the area with a duty cycle of $24 \%$ has a ( $1-$ transmission) $\approx$ absorption of $33 \%$, taken from RCWA simulations. (d) RCWA simulations of the absorption of grating-graphene structures as a function of Fermi energy and duty cycle, predicting higher absorption and thus higher field-enhancement factor $M$ for higher duty cycle. The increasing trend with Fermi energy at moderate duty cycles comes from the intrinsically larger $\mathrm{THz}$ absorption when there are more graphene charges to be accelerated.

sublinear behavior starts to play a role when the carrier temperature increase $\Delta T$ is larger than the initial carrier, or lattice, temperature $(300 \mathrm{~K})$. We note that it is the increase in carrier temperature that gives rise to the $\mathrm{THz}$ nonlinearity. ${ }^{16,22}$ Thus, the sublinear scaling of carrier temperature with light intensity gives rise to a less strong nonlinearity.

There are also saturation effects that are not captured by our thermodynamic model, thus leading to a discrepancy between experimental data points and the results of the thermodynamic model (see the blue and red shaded areas in Figure 2). We first discard the occurrence of light-induced damage to the grating or the graphene, as we did not observe any nonreversible effects of the generated harmonic signal when increasing and decreasing incident field strength, and confirmed that there was no damage by inspection with an optical microscope. We note that the damage observed in ref 13 inside their split-ring resonator gap occurred for a peak field strength of $\sim 4 \mathrm{MV} / \mathrm{cm}$, an order of magnitude larger than our highest local field inside the grating gap, which was $M \times 60=300 \mathrm{kV} / \mathrm{cm}$. Having discarded any damage, we consider saturation related to the carrier temperature of graphene that goes beyond the thermodynamic model. We point out that for the metamaterial sample the carrier temperature reaches a value above $2000 \mathrm{~K}$ already for an incident field strength of $\sim 20 \mathrm{kV} / \mathrm{cm}$ (see
Supporting Information). With heat accumulating in both the electronic and phononic systems, phonon bottleneck effects lead to a longer cooling time for the hot carrier system. We have simulated the effect of slower cooling and find that a factor 10 increase in cooling time leads to almost a factor 10 decrease in harmonic generation (see Supporting Information). Furthermore, it gives rise to a red shift of the harmonic signal, which we indeed observe (see Supporting Information). At these elevated carrier temperatures, increased phonon scattering can furthermore decrease the carrier mobility, thus giving rise to even stronger saturable absorption. Therefore, we ascribe the strong saturation effects we observe in the gratinggraphene metamaterial, even for field strengths on the order of $10 \mathrm{kV} / \mathrm{cm}$, to the comparably high carrier temperatures that we reach due to field enhancement.

Photonic Enhancement. We proceed with exploring how the intensity of the generated third-harmonic signal depends on the photonic enhancement of the metal grating and what are the design considerations to optimize the grating. First, we verify the proper operation of the grating by varying its orientation with respect to the polarization of the incident field. The measurements in Figure 1 were performed with the metal stripes perpendicular to the incoming polarization, where enhancement of field inside the gap of the grating structure is 
expected. Figure 3a shows that by rotating the sample such that the orientation of the grating with respect to the incoming $\mathrm{THz}$ polarization changes over $90^{\circ}$, the amount of generated harmonic signal is reduced to a value below our experimental noise floor. This is also as expected because, for parallel orientation of the polarization with respect to the metal stripes, no field enhancement occurs. Moreover, the grating in this case behaves as a $\mathrm{THz}$ filter that reduces $\mathrm{THz}$ transmission to the graphene film behind it.

In order to study how field enhancement depends on the duty cycle of the grating, we use a grating-graphene metamaterial sample (sample C), which contains four areas with metal gratings with different duty cycles, varying from 24 to $79 \%$ (see Figure $3 \mathrm{~b}$ ). A larger duty cycle corresponds to a smaller gap in between the metal stripes, which should lead to larger field enhancement. Figure $3 c$ shows that, with increasing duty cycle, the amount of generated harmonic indeed increases, although it saturates for the largest duty cycles. The reason that we do not see any further increase in detected third harmonic above $\eta=70 \%$ (see Figure 3c) is the occurrence of saturation effects, as discussed above. In order to gain further insights, we have performed RCWA simulations as a function of duty cycle and Fermi energy of graphene (see Figure 3d). The results show that the total amount of $\mathrm{THz}$ absorption increases with increasing duty cycle before decreasing quickly upon approaching $\eta=100 \%$. This total absorption is the sum of the absorption of the active graphene area inside the gap of the grating and of the inactive area that is covered by metal. The field in the graphene region below the metal stripes is very small, so absorption there is negligible (see Supporting Information). For our samples with Fermi energy of $\sim 0.23 \mathrm{eV}$, we expect maximum absorption for a duty cycle of $80-90 \%$. This behavior can be understood from the tradeoff between increasing field enhancement $v s$ decreasing active graphene area with increasing duty cycle. We measured the transmitted fundamental intensity for sample $\mathrm{C}$ with varying duty cycle and used it to examine how the absorption $\approx(1-$ transmitted fundamental) scales with duty cycle (see inset of Figure $3 \mathrm{c}$ ). We confirm that the overall $\mathrm{THz}$ absorption (active plus inactive area) increases moderately with increasing duty cycle. As the active area decreases with increasing duty cycle, this indicates increasingly strong field enhancement inside the gap.

The absorption simulations suggest that a grating-graphene metamaterial, despite the small active area, can reach almost $50 \%$ absorption of all incident $\mathrm{THz}$ photons onto the metamaterial for monolayer graphene with a standard mobility of $2000 \mathrm{~cm}^{2} /$ Vs. This is $\sim 3$ times more than the amount of absorption in bare graphene with a Fermi energy of $0.23 \mathrm{eV}$, as estimated for sample A (see Supporting Information). With a duty cycle of $\eta=90 \%$, that is, $10 \%$ active area, the simulations give an overall absorption of $45 \%$ in the metamaterial sample, which corresponds to an effective absorption of $450 \%$ in the active graphene area. This is a photonic absorption enhancement of $\sim 26$, which corresponds to a field enhancement of $M$ $\sim 5$ inside the gap, which is in good agreement with the electric field simulations in the Supporting Information. For the lowest duty cycle, we studied experimentally, $\eta=24 \%$, the overall $\mathrm{THz}$ absorption is still around $30 \%$. However, the field enhancement $M$ inside the gap is only $\sim 1.5$. Thus, in order to maximize the nonlinear susceptibility, the grating-graphene metamaterial would have a duty cycle approaching-without reaching-100\%. The second parameter to optimize is the
Fermi energy of graphene, where we note that a smaller Fermi energy means less intrinsic $\mathrm{THz}$ absorption. Our simulations indicate that the combination of graphene with a Fermi energy approaching the Dirac point and a metal grating with a duty cycle approaching $100 \%$ still leads to absorption close to $50 \%$. As an outlook of what is possible with our nonlinear gratinggraphene metamaterial platform, we calculate the expected nonlinearity for a grating with a duty cycle of $99 \%$ and graphene with a Fermi energy of $0.1 \mathrm{eV}$. Such a duty cycle can be achieved using a gap size of $w_{\text {gap }}=200 \mathrm{~nm}$ and $\left(w_{\text {metal }}+\right.$ $\left.w_{\text {gap }}\right)=20 \mu \mathrm{m}$. This is possible using nanofabrication techniques, such as electron beam lithography or focused ion beam milling. We estimate that we can reach $M=30$ for $\eta=$ $99 \%$ and $E_{\mathrm{F}}=0.1 \mathrm{eV}$. This would increase the third-harmonic intensity by a factor $M^{6} \times(1-\eta) \approx 10^{7}$ and increase the third-order THz nonlinearity to above $10^{-6} \mathrm{~m}^{2} / \mathrm{V}^{2}$.

Overall Conversion Efficiency and Ninth-Harmonic Generation. In addition to optimizing the nonlinear susceptibility, as we just described, an important goal toward applications is to obtain a large nonlinear conversion efficiency for relatively small incident field strength. We therefore test sample A (with $90 \%$ duty cycle) using an experimental geometry where we directly obtain the conversion efficiency. To this end, we use a table-top $\mathrm{THz}$ source, producing a moderate peak field strength of $\sim 30 \mathrm{kV} / \mathrm{cm}$. Briefly (see Methods for details), we use an amplified Ti:sapphire laser system incident on a $\mathrm{LiNbO}_{3}$ nonlinear crystal, together with two $0.3 \mathrm{THz}$ band-pass filters, to create a multicycle $\mathrm{THz}$ waveform in a narrow band around $0.3 \mathrm{THz}$. As a detection crystal, we use a $100 \mu \mathrm{m}$ thick $\mathrm{ZnTe}$ crystal with $\langle 110\rangle$ cut on top of $2 \mathrm{~mm}\langle 100\rangle \mathrm{ZnTe}^{36}$ This gives us a flat spectral response and eliminates reflections, meaning that the sensitivities for the fundamental at $0.3 \mathrm{THz}$ and the third harmonic at $0.9 \mathrm{THz}$ are identical. Crucially, we measure the transmitted $\mathrm{THz}$ waveform without any filters behind the sample, thus transmitting both fundamental and created harmonic signal. The Fourier transform is shown in Figure $4 \mathrm{a}$ and clearly contains both fundamental fields at $0.3 \mathrm{THz}$ and third harmonic at $0.9 \mathrm{THz}$. We directly obtain the ratio between generated third-harmonic and transmitted fundamental fields and find a value of $1.6 \%$, which-after correcting for an estimated $\sim 45 \%$ absorption of $\mathrm{THz}$ fundamental in the metamaterial-corresponds to a nonlinear conversion efficiency of $\sim 1 \%$. This is a highly encouraging value for the field conversion efficiency of $\mathrm{THz}$ third-harmonic generation using such a modest field strength $(\sim 30 \mathrm{kV} / \mathrm{cm})$.

As a final experiment, we examine harmonics beyond the third harmonic. As grating-induced field enhancement increases harmonic generation in a nonlinear way (see eq 1 ), this effect will be even more pronounced for higher harmonics. Analytically, we would expect the intensity of the $n=$ ninth harmonic, for example, to be enhanced by 11 orders of magnitude using our grating with a duty cycle of $90 \%$ and fieldenhancement factor $M=5$. This, however, does not take any saturation effects into consideration. Therefore, we obtain a more accurate estimate using the thermodynamic split-step calculations of $\mathrm{THz}$ harmonic generation following ref 16 . We use a modest incident field strength of $30 \mathrm{kV} / \mathrm{cm}$ in order to avoid the occurrence of saturation effects that are not captured by our model. The simulation results in Figure $4 \mathrm{~b}$ predict that the ninth-harmonic intensity for our grating-graphene nonlinear metamaterial will be enhanced by more than 7 orders of magnitude compared to that of bare graphene. To verify this 

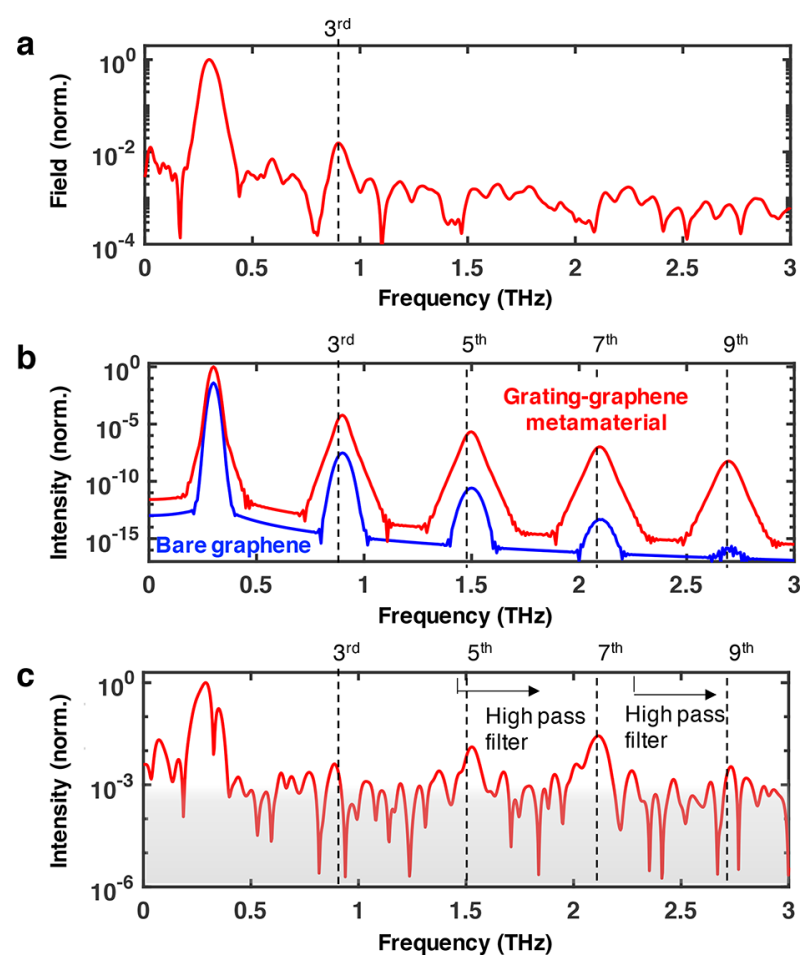

Figure 4. Conversion efficiency and ninth harmonic. (a) Spectral content of the $\mathrm{THz}$ waveform after grating-graphene metamaterial sample A using a table-top source with $30 \mathrm{kV} / \mathrm{cm}$ at $0.3 \mathrm{THz}$. We did not use any spectral filters in the detection pulse, thus allowing the amount of transmitted fundamental fields and generated harmonic in the spectrum to be quantitatively observed, giving a field conversion efficiency of $\sim 1 \%$. (b) Split-step simulation results of the effect of a grating-induced enhancement factor $M=5$ on the possibility to observe higher harmonics. The incident field is $\mathbf{3 0}$ $\mathrm{kV} / \mathrm{cm}$ at $0.3 \mathrm{THz}$. The blue (red) line is without (with) field enhancement. At the fundamental frequency, the intensity is enhanced by $M^{2}=25$, whereas, due to the higher-order nonlinear processes that give rise to higher harmonics, field confinement leads to even larger enhancement of harmonic intensity, for example, around 7 orders of magnitude for the ninth harmonic. (c) Experimental results using our table-top source with incident field strength of $\sim 30 \mathrm{kV} / \mathrm{cm}$ and grating-graphene metamaterial sample A. We put high-pass filters in the detection path in order to focus on higher harmonics, thus suppressing strongly the fundamental and third harmonic. We observe very clear signatures of the fifth and seventh harmonic and even the ninth harmonic. The gray shaded area indicates the estimated experimental noise floor.

experimentally, we use our table-top setup, now with two highpass filters behind sample A and a GaP detection crystal, which allows for a larger acceptance $\mathrm{THz}$ spectral bandwidth, as compared to the previously used $\mathrm{ZnTe}$ crystal. Figure $4 \mathrm{c}$ shows the obtained spectrum with very clear fifth and seventh harmonic. There is even a discernible signature of the ninth harmonic just above our estimated noise floor. We remark that the observation of ninth harmonic signal with incident light in the near-infrared in the strongly nonlinear epsilon near zero material $\mathrm{CdO}$ required a much larger field strength, namely, $>1$ $\mathrm{MV} / \mathrm{cm}^{37}$ This highlights the exceptional strength of the $\mathrm{THz}$ nonlinearity of our material system.

\section{CONCLUSION}

We have demonstrated a grating-graphene metamaterial, where grating-induced field enhancement significantly increases the already large $\mathrm{THz}$ nonlinearity of graphene. For a grating with a duty cycle of $90 \%$, we have obtained an effective nonlinear susceptibility $\chi_{\text {eff }}^{(3)}$ as high as $-3 \times 10^{-8} \mathrm{~m}^{2} / \mathrm{V}^{2}$ and a thirdharmonic generation conversion efficiency of $\sim 1 \%$ using a moderate incident field strength of $\sim 30 \mathrm{kV} / \mathrm{cm}$. We qualitatively understand the occurrence of this large nonlinearity as being the result of the incident field being enhanced inside the gaps of the grating. Due to the nonlinear nature of harmonic generation, the intensity of the third-harmonic signal is increased by a factor much larger than the field enhancement. Quantitatively, we find agreement using a fieldenhancement factor of 5, as obtained from RCWA calculations, and calculating harmonic generation analytically in the perturbative regime and numerically using a thermodyamic model of the $\mathrm{THz}$ nonlinearity beyond the perturbative regime. We have shown that, for incident field strengths above $\sim 10 \mathrm{kV} / \mathrm{cm}$, saturation effects related to the high carrier temperature occur. Low incident field strengths are in any case most relevant, as commercial applications require low power consumption. Based on our understanding of the metal grating, we have shown perspectives for further improving the nonlinear conversion efficiency of the grating-graphene system, in particular, by increasing the duty cycle and decreasing the Fermi energy. Finally, we have shown that the nonlinear enhancement allows for creating higher harmonics (up to the ninth). The grating-graphene metamaterial that we have introduced here relies on standard, CMOS-compatible fabrication technology using chemical vapor deposition (CVD)-grown graphene and has a very small material footprint and sufficiently high nonlinear conversion efficiency to ensure low power consumption. We thus conclude that it is an outstanding platform for commercially viable, chip-integrated, $\mathrm{THz}$ nonlinear conversion applications.

\section{METHODS}

Sample Fabrication. All samples were prepared starting with 1 $\mathrm{cm} \times 1 \mathrm{~cm}$ Infrasil quartz substrates with excellent $\mathrm{THz}$ transparency. For samples $A$ and $B$, we then used standard poly(methyl methacrylate) (PMMA)-assisted wet transfer of CVD-grown graphene (Grolltex) of roughly $1 \mathrm{~cm} \times 1 \mathrm{~cm}$. This was followed by atomic layer deposition (ALD) of $2 \mathrm{~nm}$ of $\mathrm{Al}_{2} \mathrm{O}_{3}$. For sample A, we then used optical lithography and thermal evaporation of $50 \mathrm{~nm}$ of gold with titanium as the sticking layer. Samples A and B, which are used for comparing the grating-induced harmonic enhancement, were prepared concurrently, under identical conditions with the exact same source materials, thus ensuring that their key properties-Fermi energy and mobility-are nearly identical. For sample $\mathrm{C}$, we first fabricated the metal grating, followed by ALD of $2 \mathrm{~nm}$ of $\mathrm{Al}_{2} \mathrm{O}_{3}$ and then standard PMMA-assisted wet transfer of CVD-grown graphene. The grating areas had a typical size of about $5 \mathrm{~mm} \times 5 \mathrm{~mm}$, that is, significantly larger than the spot size. See Supporting Information for a schematic overview of the samples and Raman characterization of sample B. We note that RCWA calculations show no significant difference between samples where the graphene is placed on top or below the metal grating (see Supporting Information).

Experimental Setups. Measurements were all performed at the TELBE facility at Helmholtz-Zentrum Dresden-Rossendorf, at room temperature and ambient conditions. ${ }^{28}$ See Supporting Information for a schematic of the experimental setup. The super-radiant source was tuned to provide multicycle, monochromatic, linearly polarized $\mathrm{THz}$ pulses with a frequency of $0.7 \mathrm{THz}(20 \% \mathrm{fwhm}$ bandwidth $)$ at a repetition rate of $100 \mathrm{kHz}$. The field strength was tuned using a pair of wiregrid polarizers. The incident $\mathrm{THz}$ light was focused down to a spot size with a fwhm of $870 \mu \mathrm{m}$. We used two band-pass filters at 2.1 $\mathrm{THz}$ behind the sample to reduce the transmitted fundamental field. 
The transmitted $\mathrm{THz}$ waveform, containing mainly re-emitted thirdharmonic signal, was detected with electro-optic sampling (EOS) in a $1.9 \mathrm{~mm}$ thick $\mathrm{ZnTe}$ nonlinear crystal using a synchronized Ti:sapphire laser system with 100 fs pulses. ${ }^{38}$ In the analysis of the transmitted signals, we took into account the properties of the two filters, as well as the wavelength-dependent EOS response of $\mathrm{ZnTe}$. For more details, see ref 16 .

For the measurements in Figure 4, we used a Ti:sapphire amplified laser system to pump a nonlinear $\mathrm{LiNbO}_{3}$ crystal under a tilted pulse front configuration ${ }^{39}$ to generate $\mathrm{THz}$ radiation with a broadband, linearly polarized $\mathrm{THz}$ waveform at a repetition rate of $1 \mathrm{kHz}$. We then used a band-pass filter at $0.3 \mathrm{THz}$ to create a spectrally more narrow, multicycle $\mathrm{THz}$ waveform. We detected the transmitted $\mathrm{THz}$ radiation in Figure 4a, without passing through any filters, using a 100 $\mu \mathrm{m}$ thick ZnTe crystal with $\langle 110\rangle$ cut on top of $2 \mathrm{~mm}\langle 100\rangle \mathrm{ZnTe}^{36}$ For the data in Figure 4c, we performed EOS using a $300 \mu \mathrm{m}$ thick $\mathrm{GaP}$ crystal, and we added two high-pass filters with cutoff frequencies at 1.4 and $2.2 \mathrm{THz}$ in order to resolve higher harmonics.

Split-Step Time-Domain Simulation of Thermodynamic $\mathrm{THz}$ Nonlinearity. We treat the nonlinear time-dependent response of graphene interacting with an incident $\mathrm{THz}$ field as described in detail in refs 16, 22, and 23. Briefly, the split-step approach consists of calculating the response of the system in very small temporal steps, in our case, steps of $1 \mathrm{fs}$. After the arrival and absorption of the first part of the $\mathrm{THz}$ pulse by graphene, the amount of heat contained in the electronic system increases. This corresponds to an increased electron temperature, which is associated with a lower $\mathrm{THz}$ conductivity, that is, lower $\mathrm{THz}$ absorption. This therefore affects the interaction between graphene and the next part of the incoming $\mathrm{THz}$ pulse. At the same time, the electronic system also starts to cool down, thus increasing again the $\mathrm{THz}$ absorption. These $\mathrm{THz}$-induced heatingcooling cycles of the electronic system, which lead to a temporal modulation of the $\mathrm{THz}$ absorption, are the basis of the thermodynamic $\mathrm{THz}$ nonlinearity. In our split-step simulation, we repeat the calculation of the system response every femtosecond in a looped numerical fashion. This allows us to obtain the buildup of the heat function in time and extract the transmitted field until the whole driving $\mathrm{THz}$ field is propagated through the graphene layer. Finally, we use Fourier transformation to obtain the fundamental and harmonic components of the transmitted field. We use cooling dynamics with characteristic time scales in the picosecond regime, as in refs 16 and 23. We furthermore apply a phenomenological parametrization of the effect of the amount of absorbed heat in the electronic system on the $\mathrm{THz}$ conductivity (and thus absorption), as in refs 16 and 23.

Calculation of Nonlinear Refractive Index. For Kerr-like optical nonlinearities, the nonlinear refractive index is given by ${ }^{1}$

$$
n_{2}=\frac{3 \chi_{\mathrm{eff}}^{(3)}}{4 n_{3 \mathrm{f}}^{2} \epsilon_{0} c} \text { for }\left|n_{2}\right| I_{\mathrm{THz}} / n_{0} \ll 1
$$

Here, $\epsilon_{0}$ is the vacuum permittivity. Using our $\chi_{\text {eff }}^{(3)}$ of $-3 \times 10^{-8} \mathrm{~m}^{2} /$ $\mathrm{V}^{2}$ and $n_{3 \mathrm{f}}=10$, we obtain an $n_{2}$ close to $-10^{6} \mathrm{~cm}^{2} / \mathrm{GW}$. This number is valid for a very small incident field strength. We note that even with a field strength of $5 \mathrm{kV} / \mathrm{cm}$ (equivalent to an intensity of $I_{\mathrm{THz}}=c \epsilon_{0} n \mid$ $\left.E\right|^{2} / 2 \approx 10^{-5} \mathrm{GW} / \mathrm{cm}^{2}$ ), we do not meet the criterion above. Therefore, we make an alternative estimate of $n_{2}$ that is valid for the experimentally used field strengths. We consider a field strength of $\sim 50 \mathrm{kV} / \mathrm{cm}$, where the third-harmonic signal is strongly saturated, indicating that the carrier temperature is so high that the $\mathrm{THz}$ conductivity is close to zero (see also refs 16 and 22). This means that the refractive index has decreased from $\sim 10$ to something close to 2.5 - the refractive index of graphite. ${ }^{40}$ We thus use $n_{2}=\left(n-n_{0}\right) / I_{\mathrm{TH} z}$, with $\left(n-n_{0}\right)=-7.5$ and $I_{\mathrm{THz}}=0.025 \mathrm{GW} / \mathrm{cm}^{2}$ (for a field strength of $50 \mathrm{kV} / \mathrm{cm}$ ), obtaining $n_{2}=-300 \mathrm{~cm}^{2} / \mathrm{GW}$. So the nonlinear refractive index for our experimental conditions is $\left|n_{2}\right|>100 \mathrm{~cm}^{2} / \mathrm{GW}$.

\section{ASSOCIATED CONTENT}

\section{Supporting Information}

The Supporting Information is available free of charge at https://pubs.acs.org/doi/10.1021/acsnano.0c08106.

Figures of schematic of experimental $\mathrm{THz}$ harmonics setups, data analysis with window function, sample description and characterization, grating-graphene metamaterial $v s$ bare metal grating, RCWA simulations of field enhancement, RCWA simulations of absorption enhancement, saturation effects, effect of cooling dynamics, fundamental and harmonic signals in frequency domain (PDF)

\section{AUTHOR INFORMATION}

\section{Corresponding Authors}

Sergey Kovalev - Helmholtz-Zentrum Dresden-Rossendorf, Dresden 01328, Germany; Email: s.kovalev@hzdr.de

Klaas-Jan Tielrooij - Catalan Institute of Nanoscience and Nanotechnology (ICN2), BIST \& CSIC, Bellaterra

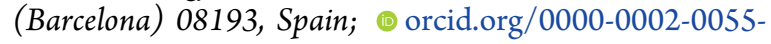
6231; Email: klaas.tielrooij@icn2.cat

\section{Authors}

Jan-Christoph Deinert - Helmholtz-Zentrum DresdenRossendorf, Dresden 01328, Germany; 이이이.org/00000001-6211-0158

David Alcaraz Iranzo - ICFO - Institut de Ciències Fotoniques, The Barcelona Institute of Science and Technology, Castelldefels (Barcelona) 08860, Spain

Raúl Pérez - Catalan Institute of Nanoscience and Nanotechnology (ICN2), BIST \& CSIC, Bellaterra (Barcelona) 08193, Spain

Xiaoyu Jia - Max-Planck-Institut für Polymerforschung, Mainz 55128, Germany

Hassan A. Hafez - Fakultät für Physik, Universität Bielefeld, Bielefeld 33615, Germany

Igor Ilyakov - Helmholtz-Zentrum Dresden-Rossendorf, Dresden 01328, Germany

Nilesh Awari - Helmholtz-Zentrum Dresden-Rossendorf, Dresden 01328, Germany

Min Chen - Helmholtz-Zentrum Dresden-Rossendorf, Dresden 01328, Germany

Mohammed Bawatna - Helmholtz-Zentrum DresdenRossendorf, Dresden 01328, Germany

Alexey N. Ponomaryov - Helmholtz-Zentrum DresdenRossendorf, Dresden 01328, Germany

Semyon Germanskiy - Helmholtz-Zentrum DresdenRossendorf, Dresden 01328, Germany

Mischa Bonn - Max-Planck-Institut für Polymerforschung, Mainz 55128, Germany; (ㅇ orcid.org/0000-0001-68518453

Frank H.L. Koppens - ICFO - Institut de Ciències Fotòniques, The Barcelona Institute of Science and Technology, Castelldefels (Barcelona) 08860, Spain; ICREA - Institució Catalana de Reçerca i Estudis Avancats, Barcelona 08010, Spain; (1) orcid.org/0000-0001-9764-6120

Dmitry Turchinovich - Fakultät für Physik, Universität Bielefeld, Bielefeld 33615, Germany

Michael Gensch - Institute of Optical Sensor Systems, DLR, Berlin 12489, Germany; Institut für Optik und Atomare Physik, Technische Universität Berlin, Berlin 10623, Germany 
Complete contact information is available at:

https://pubs.acs.org/10.1021/acsnano.0c08106

\section{Notes}

The authors declare no competing financial interest.

\section{ACKNOWLEDGMENTS}

We thank David Saleta and Jake Mehew for Raman characterization and analysis, Z. Wang for assistance in the HHG experiments, and Ulf Lehnert and Jochen Teichert for technical assistance. K.-J.T. acknowledges funding from the European Union's Horizon 2020 research and innovation program under Grant Agreement No. 804349 (ERC StG CUHL) and financial support through the MAINZ Visiting Professorship. ICN2 was supported by the Severo Ochoa program from Spanish MINECO Grant No. SEV-2017-0706. Parts of this research were carried out at ELBE at the Helmholtz-Zentrum Dresden-Rossendorf e.V., a member of the Helmholtz Association. N.A., S.K., and I.I. acknowledge support from the European Union's Horizon 2020 research and innovation program under Grant Agreement No. 737038 (TRANSPIRE). X.J. acknowledges the support from the Max Planck Graduate Center with the Johannes GutenbergUniversität Mainz (MPGC).

\section{REFERENCES}

(1) Reshef, O.; De Leon, I.; Alam, M. Z.; Boyd, R. W. Nonlinear Optical Effects in Epsilon-near-Zero Media. Nat. Rev. Mater. 2019, 4, $535-551$.

(2) Kauranen, M.; Zayats, A. V. Nonlinear Plasmonics. Nat. Photonics 2012, 6, 737-748.

(3) Miller, D. A. B. Are Optical Transistors the Logical Next Step? Nat. Photonics 2010, 4, 3-5.

(4) Aouani, H.; Rahmani, M.; Navarro-Cia, M.; Maier, S. A. ThirdHarmonic-Upconversion Enhancement from a Single Semiconductor Nanoparticle Coupled to a Plasmonic Antenna. Nat. Nanotechnol. 2014, 9, 290-294.

(5) Alam, M. Z.; De Leon, I.; Boyd, R. W. Large Optical Nonlinearity of Indium Tin Oxide in its Epsilon-near-Zero Region. Science 2016, 352, 795-797.

(6) Alam, M. Z.; Schulz, S. A.; Upham, J.; De Leon, I.; Boyd, R. W. Large Optical Nonlinearity of Nanoantennas Coupled to an Epsilonnear-Zero Material. Nat. Photonics 2018, 12, 79-83.

(7) You, J. W.; Bongu, S. R.; Bao, Q.; Panoiu, N. C. Nonlinear Optical Properties and Applications of 2D Materials: Theoretical and Experimental Aspects. Nanophotonics 2018, 8, 63-97.

(8) Nie, S.; Emory, S. R. Probing Single Molecules and Single Nanoparticles by Surface-Enhanced Raman Scattering. Science 1997, 275, 1102-1106.

(9) Fan, W.; Zhang, S.; Malloy, K. J.; Brueck, S. R. J.; Panoiu, N. C.; Osgood, R. M. Second Harmonic Generation from Patterned GaAs inside a Subwavelength Metallic Hole Array. Opt. Express 2006, 14, 9570-9575.

(10) van Nieuwstadt, J. A. H.; Sandtke, M.; Harmsen, R. H.; Segerink, F. B.; Prangsma, J. C.; Enoch, S.; Kuipers, L. Strong Modification of the Nonlinear Optical Response of Metallic Subwavelength Hole Arrays. Phys. Rev. Lett. 2006, 97, 146102.

(11) Genevet, P.; Tetienne, J.-P.; Gatzogiannis, E.; Blanchard, R.; Kats, M. A.; Scully, M. O.; Capasso, F. Large Enhancement of Nonlinear Optical Phenomena by Plasmonic Nanocavity Gratings. Nano Lett. 2010, 10, 4880-4883.

(12) Cai, W.; Vasudev, A. P.; Brongersma, M. L. Electrically Controlled Nonlinear Generation of Light with Plasmonics. Science 2011, 333, 1720-1724.

(13) Liu, M.; Hwang, H. Y.; Tao, H.; Strikwerda, A. C.; Fan, K.; Keiser, G. R.; Sternbach, A. J.; West, K. G.; Kittiwatanakul, S.; Lu, J.; et al. Terahertz-Field-Induced Insulator-to-Metal Transition in Vanadium Dioxide Metamaterial. Nature 2012, 487, 345-348.

(14) Fan, K.; Hwang, H. Y.; Liu, M.; Strikwerda, A. C.; Sternbach, A.; Zhang, J.; Zhao, X.; Zhang, X.; Nelson, K. A.; Averitt, R. D. Nonlinear Terahertz Metamaterials via Field-Enhanced Carrier Dynamics in GaAs. Phys. Rev. Lett. 2013, 110, 217404.

(15) Kim, S.; Jin, J.; Kim, Y.-j.; Park, I.-y.; Kim, Y.; Kim, S.-w. HighHarmonic Generation by Resonant Plasmon Field Enhancement. Nature 2008, 453, 757-760.

(16) Hafez, H.; Kovalev, S.; Deinert, J.-C.; Mics, Z.; Green, B.; Awari, N.; Chen, M.; Germanskiy, S.; Lehnert, U.; Teichert, J.; et al. Extremely Efficient Terahertz High-Harmonic Generation in Graphene by Hot Dirac Fermions. Nature 2018, 561, 507-511.

(17) Giorgianni, F.; Chiadroni, E.; Rovere, A.; Cestelli-Guidi, M.; Perucchi, A.; Bellaveglia, M.; Castellano, M.; Di Giovenale, D.; Di Pirro, G.; Ferrario, M.; et al. Dirac Surface States in $\mathrm{Bi}_{2} \mathrm{Se}_{3}$ Topological Insulator. Nat. Commun. 2016, 7, 11421.

(18) Kovalev, S.; Deinert, J.-C.; Ilyakov, I.; Awari, N.; Chen, M.; Ponomaryov, A.; Bawatna, M.; de Oliveira, T.; Eng, L. M.; Kuznetsov, K. A.; et al. Perturbative Regime of Terahertz High-Harmonic Generation in Topological Insulators. arXiv 2020; https://arxiv.org/ abs/2006.03948 (accessed 2020-11-24).

(19) Cheng, B.; Kanda, N.; Ikeda, T. N.; Matsuda, T.; Xia, P.; Schumann, T.; Stemmer, S.; Itatani, J.; Armitage, N. P.; Matsunaga, R. Efficient Terahertz Harmonic Generation with Coherent Acceleration of Electrons in the Dirac Semimetal $\mathrm{Cd}_{3} \mathrm{As}_{2}$. Phys. Rev. Lett. 2020, 124, 117402.

(20) Kovalev, S.; Dantas, R. M. A.; Germanskiy, S.; Deinert, J.-C.; Green, B.; Ilyakov, I.; Awari, N.; Chen, M.; Bawatna, M.; Ling, J.; et al. Non-Perturbative Terahertz High-Harmonic Generation in the Three-Dimensional Dirac Semimetal $\mathrm{Cd}_{3} \mathrm{As}_{2}$. Nat. Commun. 2020, 11, 2451.

(21) Soavi, G.; Wang, G.; Rostami, H.; Purdie, D. G.; De Fazio, D.; Ma, T.; Luo, B.; Wang, J.; Ott, A. K.; Yoon, D.; et al. Broadband, Electrically Tunable Third-Harmonic Generation in Graphene. Nat. Nanotechnol. 2018, 13, 583-588.

(22) Mics, Z.; Tielrooij, K.-J.; Parvez, K.; Jensen, S. a.; Ivanov, I.; Feng, X.; Müllen, K.; Bonn, M.; Turchinovich, D. Thermodynamic Picture of Ultrafast Charge Transport in Graphene. Nat. Commun. 2015, 6, 7655.

(23) Hafez, H. A.; Kovalev, S.; Tielrooij, K.-J.; Bonn, M.; Gensch, M.; Turchinovich, D. Terahertz Nonlinear Optics of Graphene: From Saturable Absorption to High-Harmonics Generation. Adv. Opt. Mater. 2020, 8, 1900771.

(24) Jensen, S.; Mics, Z.; Ivanov, I.; Varol, H.; Turchinovich, D.; Koppens, F.; Bonn, M.; Tielrooij, K. Competing Ultrafast Energy Relaxation Pathways in Photoexcited Graphene. Nano Lett. 2014, 14, 5839-5845.

(25) Tielrooij, K.; Song, J.; Jensen, S.; Centeno, A.; Pesquera, A.; Zurutuza Elorza, A.; Bonn, M.; Levitov, L.; Koppens, F. Photoexcitation Cascade and Multiple Hot-Carrier Generation in Graphene. Nat. Phys. 2013, 9, 248-252.

(26) Tomadin, A.; Hornett, S.; Wang, H.; Alexeev, E.; Candini, A.; Coletti, C.; Turchinovich, D.; Kläui, M.; Bonn, M.; Koppens, F.; et al. The Ultrafast Dynamics and Conductivity of Photoexcited Graphene at Different Fermi Energies. Sci. Adv. 2018, 4, No. eaar5313.

(27) Goossens, S.; Navickaite, G.; Monasterio, C.; Gupta, S.; Piqueras, J. J.; Pérez, R.; Burwell, G.; Nikitskiy, I.; Lasanta, T.; Galán, T.; et al. Broadband Image Sensor Array Based on Graphene-CMOS Integration. Nat. Photonics 2017, 11, 366-371.

(28) Green, B.; Kovalev, S.; Asgekar, V.; Geloni, G.; Lehnert, U.; Golz, T.; Kuntzsch, M.; Bauer, C.; Hauser, J.; Voigtlaender, J.; et al. High-Field High-Repetition-Rate Sources for the Coherent $\mathrm{THz}$ Control of Matter. Sci. Rep. 2016, 6, 22256.

(29) Ganeev, R. A.; Kulagin, I. A.; Ryasnyansky, A. I.; Tugushev, R. I.; Usmanov, T. Characterization of Nonlinear Optical Parameters of KDP, $\mathrm{LiNbO}_{3}$ and BBO Crystals. Opt. Commun. 2004, 229, 403-412.

(30) Vermeulen, N.; Castello-Lurbe, D.; Khoder, M.; Pasternak, I.; Krajewska, A.; Ciuk, T.; Strupinski, W.; Cheng, J.; Thienpont, H.; Van 
Erps, J. Graphene's Nonlinear-Optical Physics Revealed through Exponentially Growing Self-Phase Modulation. Nat. Commun. 2018, 9, 2675.

(31) Sirtori, C.; Capasso, F.; Sivco, D. L.; Cho, A. Y. Giant, Triply Resonant, Third-Order Nonlinear Susceptibility $\chi_{3 \omega}^{(3)}$ in Coupled Quantum Wells. Phys. Rev. Lett. 1992, 68, 1010-1013.

(32) Markelz, A. G.; Gwinn, E. G. Nonlinear Response of QuantumConfined Electrons in Nonparabolic Subbands. J. Appl. Phys. 1996, $80,2533-2535$.

(33) Kono, J.; Su, M. Y.; Inoshita, T.; Noda, T.; Sherwin, M. S.; Allen, S. J.; Sakaki, H. Resonant Terahertz Optical Sideband Generation from Confined Magnetoexcitons. Phys. Rev. Lett. 1997, 79, 1758-1761.

(34) Zanotto, S.; Tredicucci, A. Universal Lineshapes at the Crossover between Weak and Strong Critical Coupling in FanoResonant Coupled Oscillators. Sci. Rep. 2016, 6, 24592.

(35) Graham, M. W.; Shi, S.-F.; Ralph, D. C.; Park, J.; McEuen, P. L. Photocurrent Measurements of Supercollision Cooling in Graphene. Nat. Phys. 2013, 9, 103-108.

(36) Turchinovich, D.; Dijkhuis, J. I. Performance of Combined < $100>-<110>$ ZnTe Crystals in an Amplified THz Time-Domain Spectrometer. Opt. Commun. 2007, 270, 96-99.

(37) Yang, Y.; Lu, J.; Manjavacas, A.; Luk, T. S.; Liu, H.; Kelley, K.; Maria, J.-P.; Runnerstrom, E. L.; Sinclair, M. B.; Ghimire, S.; Brener, I. High-Harmonic Generation from an Epsilon-near-Zero. Nat. Phys. 2019, 15, 1022-1027.

(38) Kovalev, S.; Green, B.; Golz, T.; Maehrlein, S.; Stojanovic, N.; Fisher, A. S.; Kampfrath, T.; Gensch, M. Probing Ultra-Fast Processes with High Dynamic Range at 4th-Generation Light Sources: Arrival Time and Intensity Binning at Unprecedented Repetition Rates. Struct. Dyn. 2017, 4, 024301.

(39) Yeh, K.-L.; Hoffmann, M. C.; Hebling, J.; Nelson, K. A. Generation of $10 \mu \mathrm{J}$ Ultrashort Terahertz Pulses by Optical Rectification. Appl. Phys. Lett. 2007, 90, 171121.

(40) Blake, P.; Hill, E. W.; Castro Neto, A. H.; Novoselov, K. S.; Jiang, D.; Yang, R.; Booth, T. J.; Geim, A. Making Graphene Visible. Appl. Phys. Lett. 2007, 91, 063124. 